\section{NOTAS}

1. A. González Bueno \& V.J. Rico. Acta Bot. Malacitana, 16(1): 141-148. 1991.

2. J.Pavón aE. Acharius. Madrid, 11-VIII-1804.Uppsala Universitetsbibliotek (Handskriftsavdelningen), s/c [Agradecemos a Anna Maria Albrektson las facilidades dadas para el acceso a este documento].

3. Publicada en Mem. Real Acad. Méd. Madrid, 1: 191-204. 1797.

4. El tomo IV de la Flora peruviana et Chilensis fue publicado, entre 1954 y 1957, por E. Álvarez López (Anales Inst. Bot. Cavanilles, 12(1): 113 195; 13: 5-70; 14: 717-784; 15: 115-241).
5. R. Rodríguez Nozal. La "Oficina de la Flora Americana" (1788-1835) y la marginación del proyecto de las expediciones botánicas ilustradas. [Tesis doctoral]. [Madrid: U.C.M., 1993]

6. R. Rodríguez Nozal. Acta Bot. Malacitana, 18: 283. 1993; R. Rodríguez Nozal. Llull, 17: 403 436. 1994.

Aceptado para su publicación en Abril de 1998

Dirección del autor: Departamento de Farmacia y Tecnología Farmacéutica (Cátedra de Historia de la Farmacia y Legislación Farmacéutica). Facultad de Farmacia. Universidad Complutense. 28040Madrid.

\title{
29. EL CATÁLOGO DE LAS PLANTAS DEL PIRINEO CENTRAL. UNA OBRA INÉDITA DE CUSTODIO DEL CAMPO GARCÍA (1830-1891)
}

\author{
Antonio GONZÁLEZ BUENO y Daniel SÁNCHEZ-MATA
}

Nacido en Bielsa (Huesca), Custodio del Campo cursó estudios de Farmacia en Barcelona donde se licenció en 1854; ejerció su profesión en su localidad natal, alternándola con el estudio de la flora local; integrado en el grupo liderado por Francisco Loscos desde 1867, trabó luego contactos con la Sociedad Linneana Matritense. En 1882 obtuvo el nombramiento de ayudante interino de prácticas en la Cátedra de Historia Natural de la Facultad de Ciencias de la Universidad de Zaragoza, ciudad en la que habría de fallecer en $1891^{1}$.

No conocemos obra impresa de este botánico, salvo los datos florísticos que $\mathrm{F}$.
Loscos incluyera en su Tratado de Plantas de Aragón ${ }^{2}$; pero sí nos ha llegado alguna obra manuscrita. Entre los fondos pertenecientes a la Sociedad Linneana Matritense, hoy depositados en el Departamento de Biología Vegetal II (Facultad de Farmacia) de la Universidad Complutense de Madrid, se conservan, reunidos en un solo cuerpo, cinco manuscritos bajo el título genérico de "Catálogo de las Plantas del Pirineo Central”, debidos a la mano de Custodio del Campo. Componen la serie 40 cuartillas, rayadas a mano, donde se listan, en dos columnas, un total de 1.123 especies que constituyen el "Catálogo de la 
provincia de Huesca", confeccionado por este botánico entre los años 1864 y $1879^{3}$.

Estos escritos, salvo el primero, preliminar y de carácter general, incluyen materiales para una futura "Flora Oscense" ${ }^{4}$. C. Campo presenta a la Sociedad Linneana sus catálogos de acuerdo con la siguiente contribución:

I. "Plantas de la Provincia de Huesca / por I Custodio Campo García. Licenc do. En Farmacia / Años 1864 a 1879." Madrid, 20 de noviembre de 1880.3 págs. Tras una corta introducción, donde se insiste en las dificultades encontradas para dar a las prensas este catálogo florístico, un problema al uso entre los botánicos de la segunda mitad del siglo $\mathrm{XIX}^{5}$, presenta una pequeña guía para herborizaciones en el Pirineo Central y sugiere excursiones e itinerarios.

II. "Alto Aragón. Prova de Hues[ca] / Catálogo de las Plantas de Bielsa / Pirineos centrales." 24 págs. Un catálogo de 1.024 fanerógamas y 66 criptógamas, ordenadas siguiendo el sistema de A.P. De Candolle, y al que acompaña un resumen donde se relaciona el número de géneros, ordenados por familias, del total de su "Flora Oscense"; por tanto, un compendio de lo listado en este manuscrito más lo herborizado para la flora de Fiscal.

III. "Indicaciones de las localidades en que crecen las [plantas] Bielsetanas que á continuación se expresan / Aragon. Pirineos Centrales. Prova ${ }^{a}$ Hu[esca]." 4 págs. Una relación, esta vez no listada, de los táxones herborizados en Huesca y que, en opinión del autor, tienen especial interés; son 150 especies, presumiblemente las mismas presentadas a la Exposición Farmacéutica Nacional de $1882^{6}$. Los comentarios publicados por F. Loscos en su artículo "Plantas de D. Custodio Campo García" se corresponden, tanto en las especies como en las localidades, a las reseñadas en este manuscrito ${ }^{7}$.

IV. "Límite de la Región del Olivo / Plantas que son de Fiscal y no de Biel[sa]." 4 págs.
V. "Plantas de Benasque que no penetran en [Bielsa] (...) Plantas de Bleucu[a y] Sesa, que no se hall[an en] Bielsa. Año 1879." 4 págs. Ambos escritos representan un primer intento de aproximación fitogeográfica al conocimiento del Pirineo, se encuadra en la nueva línea de trabajo propugnada por B. Lázaro, B. Dorronsoro y J. Gila en el seno de la Sociedad Linneana, cuyo primer fruto fue la publicación del mapa con la "Distribución geográfica de las Columníferas de la Península Ibérica" elaborado por B. Lázaro y T. Andrés Tubilla en 1882. Enlazando ambos escritos se encuentran unas "Advertencas importantes" donde C. Campo apunta, tras un estudio comparado de los catálogos, la existencia de una mayor afinidad fitogeográfica entre el Valle de Benasque y la "región del olivo y de la vid", que entre ésta y la comarca de Bielsa.

\section{NOTAS}

1. Datos biográficos tomados de F. Loscos. Tratado de plantas de Aragón, pág. 5. Madrid, 1876; R.J. Gómiz. Linneo en España: 301-312. Zaragoza, 1907 y V.M.T. [Martín Tejero, V.] Gran Enciclopedia Aragonesa, 3: 602. Zaragoza, 19801982.

2. "Custodio del Campo (...) en solo dos años me ha remitido de los Altos Pirineos la enorme cifra de 900 plantas." F. Loscos. Op. cit. nota 1, pág. 5. En el ejemplar que utilizamos, conservado en la Biblioteca del Real Jardín Botánico (Madrid) hay una corrección de este párrafo "Son 500" anotó su antiguo poseedor. El mismo F. Loscos reconocerá su error "Si causan admiración los trabajos practicados por D. Custodio del Campo en solo dos años, sube mucho de punto, sabiendo que todas las 500 plantas cogidas por él (no son 900 , como equivocadamente he dicho en un principio), todas vinieron perfectamente separadas..." (F. Loscos. Op. cit. nota 1, pág. 5).

3. Archivo del Departamento de Biología Vegetal II (Facultad de Farmacia). Universidad 
Complutense de Madrid s/c. [Arch. Biol. Veg. II. UCM](Cf. A. González Bueno. Bol. Soc. Esp. Hist. Farmacia, 38(151-152): 347-358. 1987).

4. "Habiendo intentado publicar una Flora de las Plan[tas] Bielsetano-Fiscalesas con el fin de que [tu]vieran estos trabajos para la formación de la Flora [Os]cense, á mi pesar y por causas de salud no he podi[do cum]plir mis deseos, confiando que algun dia pueda verlos [rea]lizados. Entre tanto mi deber me obliga á presentar á la Sociedad Linneana Matritense el presente Catá[logo] de las plantas de Bielsa y Fiscal con algunas noti[cias que] puedan ser interesantes para los que inten[ten] herborizar ambas localidades." C. Campo. Ms. (Arch. Biol. Veg. II. UCM, s/c).

5. Problemas similares encontraron F. Loscos y J. Pardo para la publicación de su Serie imperfecta de las plantas aragonesas (cf. A. González Bueno. Bol. Soc. Esp. Hist. Farmacia, 35(137): 23-33. 1984).

6. Sobre esta exposición hay abundante documentación en el Archivo de la Real Academia de Farmacia de Madrid (Arch. R.A.F.), son de interés, entre otros "Exposición Farmacéutica Nacional. Donativo que entregaron al C.F.M. [Colegio de Farmacéuticos de Madrid] algunos individuos y corporaciones para ayuda a su celebración y ofrecimiento de premios." 35 fols. (Arch. R.A.F., leg. 142,6); "Exposición Farmacéutica Nacional. Correspondencia mantenida entre el C.F.M. sobre los individuos nombrados como jurados, y conferencias científicas a presentar." 11 fols. (Arch. R.A.F., leg. 142,7). "Exposición Farmacéutica Nacional. Correspondencia del C.F.M. sobre inscripciones, memorias, objetos y preparados que presentan los expositores." 100 fols. (Arch. R.A.F., leg. 142,12).
7. "Plantas de D. Custodio Campo García, premiadas en la Exposición Farmacéutica y adquiridas por el Instituto de Segunda Enseñanza de Huesca." En: F. Loscos. Tratado de Plantas de Aragón. Suplemento $6^{\circ}$, págs. 37-40.Madrid, 1886. A esta relación siguen: "Nombres que D. Custodio Campo me ha comunicado de plantas nuevas ó muy raras para la Flora de Aragón." (F. Loscos. Op. cit., págs. 40-42) y "Paquete de 51 plantas que desde Madrid me ha remitido D. Custodio Campo." (F. Loscos. Op. cit., págs. 4244). Estos textos constituyen las únicas publicaciones conocidas de la labor botánica de C. Campo García.

Aceptado para su publicación en Abril de 1998

Dirección de los autores: A. González Bueno. Dpto. de Farmacia y Tecnología Farmacéutica. Facultad de Farmacia. Universidad Complutense. 28040 Madrid. D. Sánchez Mata. Dpto. de Biología Vegetal II. Facultad de Farmacia. Universidad Complutense. 28040 Madrid. 